\title{
HIGHLIGHTS
}

\section{Coronavirus replication factories}

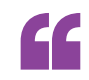

\section{a CoV}

replication

organelle com-

prising three

membrane

structures

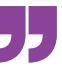

Coronaviruses (CoVs), including

severe acute respiratory syndromeCoV 2 (SARS-CoV-2), are positivestrand RNA viruses that cause respiratory and intestinal infections in humans and animals. The RNAsynthesizing machinery of CoVs associates with modified host membranes, such as double-membrane vesicles (DMVs) that are derived from membranes of the secretory pathway. As they spatially facilitate replication by concentrating proteins of the replication machinery, such modified endomembrane compartments are referred to as viral replication organelles. Moreover, several studies have implicated various additional $\mathrm{CoV}$-induced membrane structures, such as convoluted membranes, zippered ER and double-membrane spherules (DMSs) that are derived from the endoplasmic reticulum, in viral RNA (vRNA) synthesis. Therefore, it was unclear which replication organelles accommodate $\mathrm{CoV}$

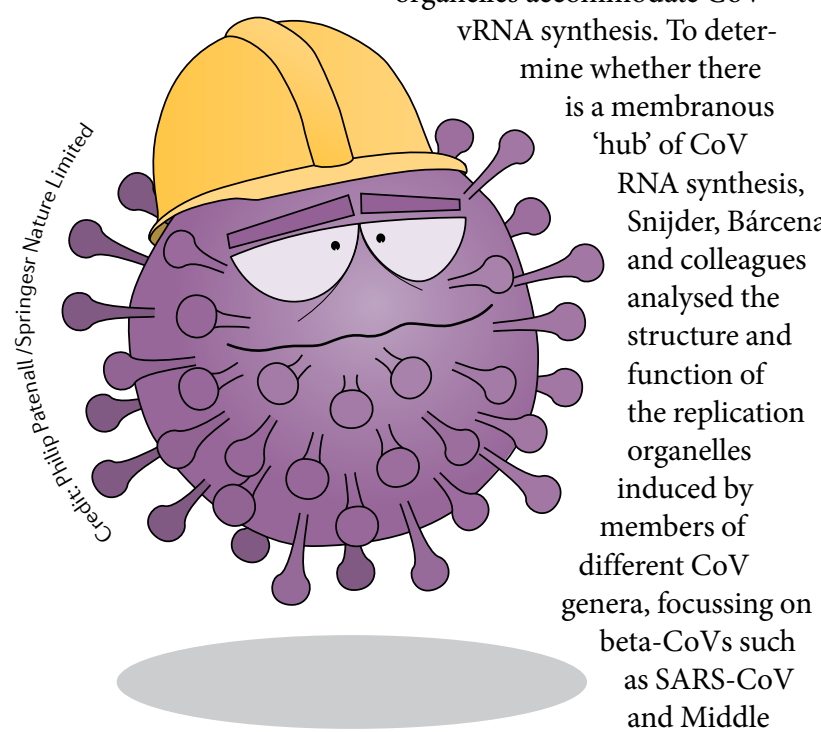

East respiratory syndrome-CoV (MERS-CoV).

First, the authors used electron tomography to show that following infection of cells, MERS-CoV induces various interconnected modified membrane structures, including convoluted membranes and DMVs, which are hallmarks of beta-CoV infections, as well as DMSs, which have not previously been reported for beta-CoVs. Moreover, the authors found that another beta- $\mathrm{CoV}$ (murine hepatitis virus), as well as a gamma-CoV (infectious bronchitis virus (IBV)) and a member of the genus alphaCoV (human coronavirus 229E) induced the same membrane structures in different cell lines. Those findings suggest that the observed clusters of modified membranes are a common characteristic of CoV-infected cells, forming the replication organelle.

Next, the authors determined the specific subcellular localization of vRNA synthesis in cells that have been infected with different $\mathrm{CoVs}$. They metabolically labelled newly synthesized vRNA followed by electron microscopy autoradiography analysis. The autoradiography signals accumulated at, and seemed to be restricted to, regions that contained virus-induced membrane modifications. This finding is in agreement with the notion that these replication organelle regions are the key areas where vRNA synthesis takes place. Importantly, DMVs exhibited dense signals in cells infected with SARS-CoV, MERS-CoV or IBV, whereas newly synthesized vRNA did not clearly associate with convoluted membranes or DMSs. These findings suggest that DMVs are the active site of CoV vRNA synthesis and that convoluted membranes or DMSs are not involved in this process.

The authors next set out to elucidate the role of convoluted membranes or DMSs in CoV replication, as they were always present in membrane-modification clusters that also contained DMVs. However, they were not able to detect proteins involved in virus assembly at DMSs or convoluted membranes, such as the membrane protein $\mathrm{M}$ and the spike protein $\mathrm{S}$, using immunoelectron microscopy, likely excluding a role in virus assembly. Future studies are needed to determine whether they represent precursor structures or whether these virus-induced structures have a specific role.

On the basis of their findings the authors propose a unifying model of $\mathrm{CoV}$ vRNA synthesis that involves the establishment of a CoV replication organelle comprising three membrane structures that are interconnected as well as connected to the ER: convoluted membranes or zippered ER, DMSs and DMVs. Within the $\mathrm{CoV}$ replication organelle, DMVs are the primary and likely only site of vRNA synthesis, which might be exploited as a potential drug target in $\mathrm{CoV}$ infection. Future studies are now needed to understand, for example, how metabolites and synthesized genomic mRNAs are transported across the double-lipid bilayer of DMVs.

Andrea Du Toit

ORIGINAL ARTICLE Snijder, E. J. et al. A unifying structural and functional model of the coronavirus replication organelle: tracking down RNA synthesis. PLoS Biol. https://doi.org/10.1371/journal.pbio.

3000715 (2020) 